Cahiers de recherches médiévales

\title{
Deux regards sur une défaite : Nicopolis
}

(d'après la Chronique du Religieux de Saint-Denis et le Livre des faits de Boucicaut)

\section{Élisabeth Gaucher}

\section{(2) OpenEdition \\ 12 Journals}

Édition électronique

URL : https://journals.openedition.org/crm/2517

DOI : $10.4000 / \mathrm{crm} .2517$

ISSN : 1955-2424

Éditeur

Honoré Champion

Édition imprimée

Date de publication : 15 janvier 1996

Pagination : 93-104

ISSN : $1272-9752$

\section{Référence électronique}

Élisabeth Gaucher, « Deux regards sur une défaite : Nicopolis », Cahiers de recherches médiévales [En

ligne], 1 | 1996, mis en ligne le 06 août 2008, consulté le 15 décembre 2022. URL : http:// journals.openedition.org/crm/2517; DOI : https://doi.org/10.4000/crm.2517 


\title{
Deux regards sur une défaite : Nicopolis (d'après la Chronique du Religieux de Saint-Denis et le Livre des faits de Boucicaut)
}

\author{
Felix qui potuit rerum cognoscere causas... (Virgile)
}

A une époque où le conflit franco-anglais et les catastrophes naturelles tendaient à estomper dans les esprits l'horizon des croisades, le péril turc réveilla la chrétienté dans sa fonction prosélytique et conquérante : l'avance de l'Infidèle, pardelà les détroits qui séparent l'Asie Mineure de l'Europe, devait être contenue. A l'appel du roi Sigismond de Hongrie, une expédition française, commandée par le fils du duc de Bourgogne, Jean de Nevers, se mit en route, bientôt rejointe par les contingents polonais et hongrois. Les croisés furent écrasés par le sultan Bajazet sur les bords du Danube, en 1396, devant les murs de Nicopolis. L'importance du désastre se mesure à l'étendue de ses répercussions. Si les témoignages ottomans présentent l'événement comme un épisode mineur, comparé aux exploits de Tamerlan en Anatolie, les sources occidentales, plus nombreuses, reflètent le traumatisme de la chrétienté.

Nous n'en retiendrons que deux, légèrement postérieures à la défaite : la Chronique de Charles VT, écrite en latin par Michel Pintoin, chantre de l'abbaye de SaintDenis, entre 1388 et 1421 , et le Livre des faits du maréchal Boucicaut, achevé en 1409 par un auteur anonyme, peut-être Nicolas de Gonesse ${ }^{1}$. D'un côté, une œuvre officielle, commandée par l'abbé de Saint-Denis pour la gloire du roi et du royaume de France, de l'autre, une biographie privée, probablement entreprise à l'instigation de Boucicaut lui-même, alors en mal de popularité. La défaite de Nicopolis n'y figure donc pas sous la même perspective. Plus que l'événement lui-même, c'est sa perception qui nous intéresse ici, sa réécriture par deux auteurs qui, conjuguant les

\footnotetext{
${ }^{1}$ Chronique du Religieux de Saint-Denys, éd. et trad. par M.L. Bellaguet, introduction de B. Guenée, Paris, Editions du Comité des travaux historiques et scientifiques, 1994 (éd. orig. 1842), livre XVII, chap. III et chap. XXIII à XXIX ; Le Livre des fais de Bouciquaut, éd. par D. Lalande, Genève, Droz, 1985 (T.L.F.,331), livre I, chap. XXII à XXVIII. Par la suite, nous abrégerons ainsi : Chron. et Bouc., sans répéter le numéro des livres; les chiffres romains renvoient aux chapitres, les chiffres arabes aux pages. Sur les auteurs de ces deux textes, voir N. Grévy-Pons et E. Omato, "Qui est l'auteur de la chronique latine de Charles VI dite du Religieux de Saint-Denis?», Bibliothèque de l'Ecole des Chartes, 134, 1976, pp. 85-102, et H. Millet, "Qui a écrit le Livre des fais de Boucicaut?», Pratiques de la culture écrite en France au $X V^{e}$ siècle (actes du colloque international du C.N.R.S., Paris, 18-21 mai 1992), éd. E. Ornato et N. Pons, Louvain-la-Neuve, 1995.
}

Cahiers de Recherches Médiévales (XIIT'-XVE s.), 1, 1996 
efforts de l'historien et du moraliste, n'en poursuivent pas moins des intentions divergentes ${ }^{2}$.

L'historien doit dire la vérité, restituer la réalité sous tous ses aspects : non seulement les belles actions, mais aussi les plus basses turpitudes. Michel Pintoin présente ainsi sa Chronique comme un diptyque, un «tableau d'honneur et d'opprobre» ${ }^{3}$, qui rassemble «tout ce qui fut digne d'éloge ou de blâme» (gesta... non modo commendabilia sed et note subjacencia scribenda). Enoncée dans le prologue, cette pétition de principe revient pour justifier le récit du désastre de Nicopolis ${ }^{4}$. Une telle déclaration dispense d'apporter d'autres garanties : à elle seule, elle signifie le refus d'enjoliver le réel. Moins assuré, le biographe de Boucicaut multiplie les précautions oratoires au début de l'épisode. Il s'agit pour lui de rétablir la «verité» sur un événement controversé, «pour cause que en plusieurs manieres et differencieusement l'une de l'autre on en devise». Il prétend répondre à la demande de «plusieurs gens» qui «desirent savoir du fait toute la maniere», et lui même trouve du plaisir («me plaist”) à raconter la bataille (XXII, 88). Tel n'est pas le cas de Michel Pintoin, qui aurait voulu l'ensevelir sous un silence éternel (perpetuo silencio) et qui se soumet aux obligations de sa charge d'historiographe officiel, non sans éprouver une grande amertume (cum cordis amaritudine, XXIII, 482), n'engageant que sa responsabilité morale, libre de toute promesse à l'égard d'un public dont l'attente risquerait d'infléchir son récit.

Pour que resplendisse la lumière de la vérité, il faut en éliminer les détails qui pourraient l'obscurcir. Michel Pintoin va droit au fait, évite les longueurs inutiles (prolixitatem declinans studiose, III, 428). Il refuse, par exemple, de sacrifier au motif du "catalogue des vaisseaux», sans doute parce qu'une liste exhaustive des participants n'aurait à ses yeux d'autre effet que de flatter les survivants de l'expédition, y compris les étrangers, peu utiles à la gloire nationale qu'entend servir le Religieux de Saint-Denis. Si le biographe de Boucicaut feint lui aussi d'éviter la prolixité, c'est pour mieux excuser l'outrance de son panégyrique : par une sorte de coquetterie qui en dit plus qu'elle n'en cache, il décide de taire les exploits de son héros, af in de ne pas s'attirer les soupçons de son lecteur par une «eslongne» semblable à celles qu'«aucuns dicteurs ont accoustumé de mettre en rommans» (XXV, 108). Or tout le Livre des faits repose sur une esthétique romanesque : pas plus que le reste de l'actualité, la bataille de Nicopolis n'échappe à cette contamination de l'histoire par la fiction.

Révélée au grand jour, épurée de ses scories, cette «vérité» n'en reste pas moins lointaine. Ni Michel Pintoin ni le biographe de Boucicaut ne se sont rendus sur les bords du Danube. Leur perspective ne dépasse pas les murs de leur chambre, ou du moins l'enceinte de Paris, puisqu'ils semblent évoquer un souvenir personnel en parlant de la consternation dans laquelle la nouvelle du désastre jeta la capitale, en

\footnotetext{
${ }^{2}$ Sur la réalité de la bataille de Nicopolis, voir A.S. Atiya, The Crusade of Nicopolis, London, 1934 ; R. Rosetti, "Notes on the Battle of Nicopolis (1396)", Slavonic and East European Review, 15, 1937, pp. 629-638 ; J. Delaville Le Roulx, La France en Orient au XIV siècle. Expéditions du maréchal Boucicaut, Paris, E. Thorin, 1886, t.I, pp. 159-165.

${ }^{3} \mathrm{~B}$. Guenée, introduction à l'éd. citée, p. L.

${ }^{4}$ Chron., livre I, I, 4 et livre XVII, XXIII, 482.
} 
décembre 1396, et des offices qui y furent célébrés à la mémoire des martyrs chrétiens $^{5}$. Mais de l'expédition elle-même, ils n'ont qu'une perception médiatisée.

Toutefois, bien que Michel Pintoin ait vécu à l'écart du monde des chevaliers, il en connaissait beaucoup, soit pour les avoir rencontrés à la cour, soit à l'occasion de leur passage à Saint-Denis ${ }^{6}$. L'un d'eux, réchappé de Nicopolis, figure explicitement dans la Chronique: Gauthier des Roches, noble bourguignon, fut un informateur précieux, que recommandaient et ses exploits et sa naissance (quidam miles actibus et genere clarus, XXVIII, 520). Pourquoi Michel Pintoin, d'ordinaire si discret sur ses sources, mentionne-t-il son nom ? Il s'agit, à la fin du récit, d'authentifier un miracle (miraculum) : longtemps après le combat, les bêtes laissèrent intacts les corps des chrétiens gisant sans sépulture, tandis qu'elle profanaient les fosses voisines où reposaient les cadavres turcs. Le spectable dérange, non seulement par son caractère merveilleux (mirabile), mais par les commentaires qu'il suscita entre Gauthier des Roches, qui y voyait l'effet de la grâce de Dieu, et le Sultan, pour qui le phénomène s'expliquait par l'impureté des chrétiens (christiani tot impietatibus pleni, XXVIII, 520). Même si l'accusation séduisait la rigueur morale du Religieux de Saint-Denis, elle présentait trop de violence pour qu'il puisse l'assumer personnellement. Mais il n'accueille pas tous les témoignages avec la même facilité : avant de citer celui de Gauthier des Roches, il prend soin de préciser qu'il a mené son enquête auprès de plusieurs personnes (pluribus inquisisse). Comme le biographe de Boucicaut, il est confronté à la multiplicité des rapports divergents, et doit faire un tri, en privilégiant "ceux qui savent lire au fond des consciences» (qui conscienciarum secretissima rimantur, XXV, 502). Les autres références aux sources occupent, elles aussi, les points stratégiques de son récit : elles forcent la croyance du lecteur, à propos d'un «acte de cruauté inouï» (le massacre des prisonniers turcs par les Français, ibid., 500), d'une estimation chiffrée écrasante (la supériorité des effectifs turcs sur les contingents alliés, XXVI, 504), des sentiments «secrets» de Bajazet (son optimisme fortifié par l'imprudence des chrétiens, ibid., 508) ou d'un spectacle pathétique (l'ultime combat de l'amiral Jean de Vienne, seul au milieu des infidèles)... Autant d'épisodes peu favorables à l'image d'une chrétienté parjure, disloquée, méprisée : de toute évidence, Michel Pintoin a du mal à avouer une vérité qui lui pèse. Si la prudence conduit aussi le biographe à déléguer son discours à des relais, appelant à la barre ceux qui participèrent à l'expédition aux côtés du maréchal et qui «le virent» $(X X V, 109)$, c'est, au contraire, pour autoriser un éloge souvent hyperbolique : et ces figures, anonymes, évoquées dans un style formulaire, renvoient plus à des stéréotypes bien connus du genre épidictique, qu'à des individus dotés d'une existence historique facilement identifiable. De fait, rien ne permet d'identifier avec précision la nature des sources. Tout comme le biographe, il semble que le Religieux de SaintDenis ait le plus souvent reçu des informations orales, à moins qu'il n'ait eu en mains des rapports rédigés à l'intention du roi ${ }^{7}$.

Or la prédominance des sources orales entraîne souvent un certain flou dans la chronologie. Le biographe de Boucicaut ne donne aucune date. Michel Pintoin, lui,

\footnotetext{
${ }^{5}$ Chron. XXIX ; Bouc. XXVII.

${ }^{6} \mathrm{~B}$. Guenée, introduction à l'éd. cit., p. XXV.

${ }^{7} \mathrm{~B}$. Guenée, introduction à l'éd. cit., p. XXXIX-XL.
} 
balise l'événement avec plus de soin : le livre XVII de sa Chronique, consacré à l'année 1396, suit la succession des mois, et la campagne de Nicopolis se déroule selon un calendrier précis, qui montre avec quelle rigueur l'enquête fut menée. Leur divergence tient à la nature même des deux textes : Michel Pintoin écrit une Chronique, tandis que le Livre des faits relève du genre biographique. D'un côté, la vérité se détache sur un fond d'histoire collective, de l'autre, elle se limite aux actions du héros. La bataille de Nicopolis acquiert une signification différente selon la perspective adoptée.

Pour Michel Pintoin, l'expédition devait servir la chrétienté tout entière (ad honorem nominis christiani, XXIII, 488). Son succès dépendait de la cohésion des croisés. Ce principe trouve un écho dans les avertissements répétés du roi de Hongrie, qui conseille à ses alliés de substituer à leurs impulsions désordonnées le respect d'une unité fondée sur les liens de la solidarité (omnes rogans humiliter ut unitatem servantes in caritatis vinculo... non dirigantur impetu, ibid., 490). Le royaume de France doit construire son prestige dans la défense du royaume de Dieu. $\mathrm{Si}$, lorsque Charles VI envoie son armée au secours de Sigismond, le calcul politique se mêle à la dévotion, puisque le roi saisit en même temps l'occasion de signaler la puissance de la France et de venger l'honneur de la chrétienté (ad strenuitatis titulum acquirendum et... ad laudem fidei christiane, ibid., 486), il n'y a pas de contradiction dans cette double postulation. Mais le comte de Nevers et les jeunes chevaliers de sa suite ont tôt fait de remplacer la raison d'Etat par la recherche de leur gloire personnelle. En confiant à son fils la tête de l'expédition, Philippe le Hardi lui offrait le moyen d'une consécration guerrière enviée de tous les «bacheliers» : «endosser ses éperons de chevalier en marchant contre les ennemis du Christ» (hunc optabat accingi baltheo militari agrediendo adversarios Crucifixi, III, 428). Dès le début, la campagne déviait vers la recherche d'un profit étranger à la vocation du très-chrétien royaume de France, et Michel Pintoin ne pouvait l'ignorer, puisque l'ambition du duc de Bourgogne pour «son fils bien aimé» s'était exprimée entre les murs mêmes de l'abbaye de Saint-Denis (Dux... ad ecclesiam beati Dyonisii dilectum adduxit filium, ibid.). Ce décalage entraîne une incompréhension permanente entre Sigismond et les compagnons de Jean de Nevers, ceux-ci refusant de suivre les conseils de prudence de celui-là, par crainte du ridicule ("hoc in opprobrium nostrum et cunctis populis in risum verteretur», XXIII, 490). Or, parmi les champions de cette «fureur guerrière» (marcius ardor), Michel Pintoin cite le nom de Boucicaut.

De fait, le biographe insiste lui aussi, mais dans une visée apologétique, sur l'impatience qui pousse son héros à occuper les devants de la scène : à peine Sigismond annonce-t-il son intention de prendre le fort de Rachowa, que le maréchal projette une «emprise pour y estre des premiers» (XXIV, 95). Lorsqu'il exhorte ses compagnons à la bataille, il flatte leur orgueil : «faisons tant en ceste besongne que il soit renom de nous» (ibid., 96). Tous leurs efforts tendent vers un même but, clairement formulé au début de l'expédition : «eulx tirer hors de oyseuse et emploier leur temps et leurs forces en fait de chevalerie» (XXII, 90). La guerre sainte s'offre comme une fête chevaleresque organisée pour occuper la jeunesse désœuvrée. Si Boucicaut, au moment de partir, n'a pas totalement oublié les motivations religieuses de l'entreprise, des préoccupations courtoises sont venues conforter son engagement, 
à savoir l'obligation de rendre service au roi de Hongrie, qui lui avait fait «bonne chere» lors de son précédent séjour, et le plaisir de suivre le comte d'Eu, «pour la grant amour que il avoit a lui qui entreprenoit le voyage» (ibid., 89). On pense à un voyage d'agrément... D'ailleurs, Sigismond a su mener une habile campagne publicitaire en annonçant «a tous bons chevaliers et escuyers qui desiroient accroistre leurs honneurs et leur vaillance» que "moult estoit le voyage honnourable» (ibid.).

La terminologie utilisée par nos auteurs fournit, en effet, une clé utile pour qui veut accéder à leur «idéologie de la croisade ${ }^{8}$. Si «la bataille de Nicopolis ne fut pas une véritable croisade, mais essentiellement une action défensive contre les Ottomans»", les noms qui la désignent sont empruntés au registre des croisades, et celui-ci repose lui-même sur l'extension, l'adoption et l'adaptation de lexiques voisins $^{10}$. Michel Pintoin reste évasif sur la nature de l'expédition, résumée dans la notion de «départ» (profectio, III, 428). En revanche, le biographe de Boucicaut alterne entre deux appellations significatives, «voyage» et «erre», qui s'emploient aussi à propos des pèlerinages ${ }^{11}$. Le premier, dérivé de via, garde une certaine ambivalence, évoquant non seulement la route mais la voie, donc l'entrée (au royaume de Dieu). Dans son acception la plus générale, il trahit l'un des facteurs d'attirance qui a assuré le succès mondain de l'expédition : voir du pays, rechercher l'exotisme. De fait, les croisés, enthousiasmés par des perspectives d'aventures lointaines, n'étaient pas toujours fixés sur la destination à suivre : les organisateurs de la campagne de Nicopolis auraient hésité entre la Prusse et la Hongrie ${ }^{12}$. Boucicaut lui-même, dans ses combats au service de Dieu, montrait une relative indifférence quant à leur localisation : s'il décida d'aller en Prusse en 1390, c'est parce que le roi lui avait interdit d'accompagner le duc de Bourbon en Tunisie ${ }^{13}$. Quant au mot «erre», qui trouve son origine dans le latin iter, il évoque un déplacement physique, lié à une ascèse douloureuse, et moins sûr de l'aboutissement final : «l'iter est franchissement d'espace, courage du corps qui avance et progressif dénuement de soi et de toutes les attaches avec la terre de la naissance ${ }^{14}$. On peut s'étonner de rencontrer ce terme à propos d'une armée dont les moralistes du temps décriaient le confort insolent... Le mélange sémantique des notions de croisade et de pèlerinage a l'avantage de masquer leur différence essentielle : la croisade est une entreprise collective, par laquelle la chrétienté manifeste sa vitalité expansive, tandis que le pèlerinage en Terre Sainte est senti comme un exercice de pénitence, inspiré par l'imitatio Christit ${ }^{15}$. Leur associa-

\footnotetext{
${ }^{8} \mathrm{Cf}$. P. Rousset, Histoire d'une idéologie : la croisade, Lausanne, éditions L'Age d'Homme, 1983.

${ }^{9}$ Ibid., p. 107.

${ }^{10}$ Le mot «croisade» n'apparaît qu'en 1460 (cf. Dictionnaire historique de la langue française, Paris, éd. Le Robert, 1992). Sur le vocabulaire de la croisade en ancien français, voir D.A. Trotter, Medieval French Literature and the Crusades (1100-1300), Genève, Droz, 1987 (chap.II).

11 "Voyage» : XXII, 89 et XXIII, 92 ; «erre» : XXIII, 92.

${ }^{12}$ N. Housley, The Later Crusades (1274-1580), Oxford University Press, 1992, p. 402.

${ }^{13}$ Bouc., XVIII, 74.
}

${ }^{14}$ A. Dupront, Du Sacré. Croisades et pèlerinages. Images et langages, Paris, Gallimard, 1987 (Bibliothèque des Histoires), pp. 257-258.

${ }^{15}$ N. Housley, op. cit., p. 401 . 
tion conduit à une complémentarité idéologique : «le pèlerinage sacralise la guerre que fait la croisade. Et dans la croisade, le pèlerinage est élevé à une réalité plus haute : il se fait acte commun... comme si la croisade portait la réalité sacrale du pèlerinage à être acte panique et glorieux du salut commun» ${ }^{16}$. Le biographe de Boucicaut recourt délibérément à un lexique valorisant pour répondre à l'accusation d'immoralité qui frappait les seigneurs engagés à Nicopolis. Ses emprunts à la terminologie du pèlerinage lui permettent aussi d'individualiser l'entreprise, et de focaliser l'éclairage sur l'engagement personnel de son héros dans le martyre de la foi. Au moment de retracer la défaite finale, il concentre sur Boucicaut son appel à l'aide divine : «Ha, Dieux! quel chevalier! Dieu lui sauve sa vertu! Dommage sera quant vie lui fauldra!» $(\mathrm{XXV}, 112)$. Or ce type d'invocation, chez le Religieux de SaintDenis, s'insère dans une perspective de rachat commun : avec une résignation qui rappelle le discours sur le Mont des Oliviers («Père, que ta volonté soit faite»), Michel Pintoin reconnaît devant le Christ lui-même le caractère abyssal de la justice cẻleste (judicia tua abyssus multa), mais voit dans le massacre des chrétiens un châtiment qui leur ouvrira, à tous, la porte de la gloire éternelle (utinam ad eorum gloriam sempiternam!, XXVII, 510-512).

A la différence de la biographie, la chronique fait rarement état des exploits individuels. Michel Pintoin n'isole que la figure de l'amiral de France, Jean de Vienne, qui porte l'étendard de la Vierge Marie. C'est lui qui exhorte les chevaliers à la résistance, tandis que le biographe attribue à Boucicaut cette preuve de courage. Mais leurs harangues ne se ressemblent pas: Jean de Vienne place toute sa confiance dans la Providence divine, alors que Boucicaut donne des conseils tactiques qui préservent une part de responsabilité humaine dans l'issue des combats ${ }^{17}$. Si Michel Pintoin distingue ainsi l'amiral de France, c'est parce qu'il porte l'emblème de la chrétienté, le signe d'une confédération de nations unies par leur foi. Loin de décrire la bravoure d'un chevalier attaché à défendre sa vie, il admire le sacrifice de Jean de Vienne, engagé corps et âme dans la cause chrétienne : assailli de tous côtés par les Turcs, l'amiral leur oppose une résistance sauvage dans laquelle il se confond avec l'image de la Vierge, abbatue et relevée six fois de suite, jusqu'à la chute finale, placée sous le symbole du chiffre sept ${ }^{18}$. A l'inverse, le biographe de Boucicaut s'attache à mettre en lumière la supériorité de son héros sur le reste des alliés : chaque "période» commence par un tableau général, brusquement interrompu par l'intervention décisive de Boucicaut. L'idéalisation du héros atteint son point culminant au chapitre XXVIII, entièrement consacré à ses démarches auprès de Bajazet pour négocier le rachat des prisonniers français et réduire le montant de leur rançon. C'est magnifier le rôle du maréchal, au détriment des ambassadeurs de Charles VI, dont la médiation fut tout aussi déterminante ${ }^{19}$. Ainsi, malgré les écarts de perspective, Michel Pintoin et le biographe de Boucicaut trahissent le même dessein : ils cherchent moins à reproduire une vérité vérifiable qu'à diffuser une vérité de foi. A l'historien s'associe le moraliste.

\footnotetext{
${ }^{16}$ A. Dupront, op. cit., p. 244.

${ }^{17}$ Chron., XXVI, 504 et XXVII, 514 ; Bouc., XXV, 106.

${ }^{18}$ Chron., XXVII, 514.

${ }^{19}$ D. Lalande, introduction à l'éd. citée, p. XXXIII.
} 
Du Religieux de Saint-Denis, Bernard Guenée écrit : «son ambition est moins de comprendre que de jugers ${ }^{20}$. Dès le début de sa Chronique, Michel Pintoin affirme qu'il ne se contentera pas d'un récit fidèle (fida relacione), mais qu'il transcrira «tout ce que la voix de sa conscience aura dicté à l'oreille de [son] cour» (que ad aurem cordis mei loquetur fides mea, livre I, I, 4). De même, l'engagement polémique du biographe de Boucicaut infléchit son rapport vers un traité de morale. Au réquisitoire de l'un répond l'apologie de l'autre, comme si leurs écrits devaient fournir des pièces à conviction au dossier judiciaire de Nicopolis.

Le Religieux de Saint-Denis ne cache pas sa colère lorsqu'il évoque les excès et l'indiscipline des jeunes nobles qui suivirent Jean de Nevers. Il fustige le luxe de leurs tentes (in tentoriis depictis), la recherche des vins et des mets les plus délicats (exquisitis vinis et dapibus), le ridicule de leurs costumes, notamment la longueur démesurée de leurs chaussures à la poulaine (calciamenta rostrata longitudinis duorum pedum et quandoque amplius), les débauches auxquelles ils se livrent en compagnie des filles de joie (omne fornicacionis genus), et la pratique des jeux de hasard (taxillorum ludo). Son indignation ressort d'autant plus violemment qu'il ménage un savant contraste entre ce tableau de perversités, dont les teintes démoniaques annoncent les peintures d'un Jérôme Bosch, et les évocations qui l'entourent : d'une part, les prières du roi de France et du clergé pour le succès des croisés, d'autre part, le dégoût de Bajazet lui-même qui, instruit des abominations perpétrées dans le camp chrétien, méprise un peuple si peu respectueux de son Dieu ${ }^{21}$. L'imminence du châtiment se devine à travers ces deux forces, celle de la justice divine et celle du péril turc, qui ressèrent leur étau sur l'armée chrétienne. Mais la colère éloigne Michel Pintoin du genre historique : aussi ferme-t-il cette parenthèse violente pour «revenir à [son] sujet» (ad propositum rediens, XXV, 499). Naturellement, le biographe de Boucicaut ne dit mot du train de vie licencieux des croisés. Tout au plus avoue-t-il que le comte de Nevers et ses hommes étaient à table quand on vint leur annoncer que les Turcs approchaient $(X X V, 103)$. Il semble désireux de taire l'insouciance des Français à la veille de la bataille, puisque Boucicaut en était largement responsable. En effet, selon Michel Pintoin, celui-ci aurait opposé la plus grande insolence aux avertissements du roi de Hongrie (in verba presumpcionis..., insolentissimis verbis, XXIII, 488-490). Il dépeint Boucicaut comme un esprit buté, obstiné, qui rend caducs les conseils des sages : c'est «jeter paroles au vent» (vento verba dare, XXIV, 492), "parler à un âne sourd» (ac si asino surdo narrassent fabulam, XXIII, 484) et perdre son temps devant un homme qui, tel l'aspic insensible, se bouche les oreilles (velut aspis surda obturans aures suas, XXIV, 494). Au contraire, le biographe insiste sur les mérites d'un chevalier qui associe à sa vaillance l'exercice de son grand «sens" (XXIV, 99; XXVIII, 124, 126, 128). Là où le Religieux de Saint-Denis condamne la folle ardeur des juniores et vante l'expérience des chevaliers aguerris (plus experti in armis, XXIII, 488), le biographe répond que l'âge ne fait rien à l'affaire chez les «nobles jouvenciaulx de la fleur de lis qui la se combatoient, non mie comme enfans, mais comme se ce fussent tres endurcis cheva-

${ }^{20} \mathrm{~B}$. Guenée, introduction à l'éd. citée, p. L.

${ }^{21}$ Chron., XXIV, 496 et 498. 
liers» (XXV, 110). Bien plus, il va jusqu'à inverser la position de Michel Pintoin sur l'obéissance due aux chefs, en attribuant à leur autorité mal placée les rares défaites attestées dans l'histoire de la chevalerie française («par la faulte de leurs chevetains", ibid., 108). Les deux récits s'opposent parfois avec une rigoureuse symétrie, par le recours aux mêmes images: ainsi, Michel Pintoin compare la retraite des chrétiens face aux Turcs à un troupeau de moutons affolés (quasi oves errantes passim per planiciem turmatim currentes vagabantur, XXVII, 512), tandis que le biographe semble reprendre terme à terme la comparaison pour mieux la nier («et est assavoir... que, sauve la grace des diseurs qui ont dit et rapporté du fait de la bataille que noz gens y fuyrent et alerent comme bestes sanz ordonnance..., et que par ce furent occis par troppiaulx au feur que ilz venoient, que ce n'est mie voir», XXV, 104). Michel Pintoin n'accorde aucune indulgence aux chrétiens. Leurs manières de combattre ne respectent pas toujours l'éthique chevaleresque, à tel point que les Turcs s'en étonnent (admirantes belli genus insolitum, XXVI, 508). Parjures, ils n'hésitent pas à égorger les vaincus auxquels ils avaient d'abord accordé la vie sauve, et ce geste leur vaudra, le lendemain, d'endurer la vengeance de Bajazet ${ }^{22}$. Or, non seulement le biographe de Boucicaut omet de signaler le massacre des prisonniers turcs à Rachowa, mais il attribue au sultan une crainte respectueuse à l'égard des chrétiens : «le Bazat fu espouenté de la grant vaillance des François» (XXV, 110). Aussi quand le possessif nostri apparaît sous la plume de Michel Pintoin pour qualifier ses compatriotes adonnés à leurs représailles (XXIV, 494: XXV, 500), le mot se charge-t-il d'une lourde ironie, bien loin de la fraternité qu'éprouve le biographe à leur égard.

Ni l'un ni l'autre ne s'en tiennent à l'exposé des faits. Leur récit laisse entendre les plaintes de deux contemporains fortement marqués par l'événement de 1396. L'histoire devient alors «tragédie»" ${ }^{23}$. Incapable d'observer l'ataraxie nécessaire à l'historien, Michel Pintoin s'abandonne souvent aux lamentations. L'épisode de $\mathrm{Ni}$ copolis s'ouvre sur un lamento où s'expriment les souffrances de l'écrivain au souvenir de ce «désastre lamentable» (discrimen lamentabile) : tiraillé entre l'amertume (amaritudine), l'effroi et la confusion (territus et confusus, XXIII, 482), le coeur offre un terrain trop agité pour construire un discours sans failles. Le Religieux de Saint-Denis pleure sur la déchéance des chrétiens : il ne peut garder les yeux secs au moment d'évoquer le traitement qu'ils réservent à leurs captifs (scribere siccis oculis non valemus, XXV, 500). Mais il pleure aussi sur leur propre «passion», sur cet événement affligeant (lugendum) dont le souvenir suscitera bien des larmes aux générations futures (ad condolendum, XXVII, 512). La description du martyre des chrétiens acquiert les dimensions d'une représentation dramatique, d'un mystère auquel Michel Pintoin nous invite à assister dans une douleur partagée (unde lacrimas non possumus continere. Quis tam ferrei pectoris, tam mentis adamantine, cujus interiora non liquefierent?, XXVIII, 516-518). De même, le biographe de Boucicaut multiplie les exclamations pathétiques au moment de retracer la mort de «tant noble compagnie» («Ha! quel dommage et quelle pitié!», XXV, 112). On peut s'interroger

\footnotetext{
${ }^{22}$ Chron, XXV, 500 ; XXVIII, 516.

${ }^{23} \mathrm{~B}$. Guenée, «Tragédie et histoire chez le Religieux de Saint-Denis», Bibliothèque de l'Ecole des Chartes, 150, 1992, pp. 223-244.
} 
sur la sincérité de ces épanchements. Par-delà l'émotion réelle des deux auteurs, impliqués dans l'événement l'un par son amour filial pour l'Eglise et la France, l'autre par son attachement au maréchal Boucicaut, ce style déclamatoire ne sert-il pas une stratégie forçant le lecteur à tirer leçon du malheur? De fait, les larmes n'expriment pas seulement la douleur, mais aussi la révolte (chez Michel Pintoin, indigné par l'aveuglement des chrétiens : o ceca mortalis infirmitas!, XXVI, 514) ${ }^{24}$, voire l'appel à la vengeance (chez le biographe de Boucicaut, à propos des traîtres : «que male honte leur puisse venir!», XXV, 112).

La bataille de Nicopolis offrait un argument de choix au didactisme. Mais si Michel Pintoin ouvre sa Chronique sur l'intention de donner au jeune Charles VI "une règle de conduite à suivre» (rerum gerendarum regulam imitandam, livre I, I, 2), c'est au service de «Chevalerie» que le biographe de Boucicaut met toute sa «Science» «affin que ceulx qui tendent a honneur puissent prendre exemple de bien faire» (I, 9). Chez l'un, on trouve donc une perspective nationale, chez l'autre, un traité de convenances sociales - miroir du prince, miroir du chevalier, à une époque où la conjoncture rend difficile la superposition des deux figures.

La France est le royaume de la Nouvelle Alliance, le peuple élu de Dieu ${ }^{25}$. Aussi, dans son récit du voyage de Hongrie, Michel Pintoin la confond-il avec la chrétienté en marche, le bouclier de la foi, et son argumentation laisse peu de place aux acteurs de sa puissance séculière. En revanche, le biographe de Boucicaut met en avant le prestige personnel du comte de Nevers et de ses hommes, «tous vaillans champions de la noble contree de François» (XXV, 107). Le Religieux de SaintDenis propose une interprétation théologique de l'événement. Les défaites militaires sont dues à l'ingratitude du peuple français, qui rompt unilatéralement la Sainte Alliance. Dieu a puni les vices des chrétiens à Nicopolis et leur a envoyé un châtiment en la personne de Bajazet ${ }^{26}$. Tel n'est pas le système causal que privilégie le biographe de Boucicaut. Pour lui, la trahison des Hongrois fut décisive, et il insiste sur leur "mauvaistié», leur «felonnie» et leur «lacheté» (XXV, 107). A l'inverse de Michel Pintoin, qui admire la sagesse de leur roi, il accuse celui-ci d'avoir joué un «mauvais tour» aux Français en les avertissant trop tard de l'avancée des Turcs (ibid., 103). La défaite s'explique donc par des causes proprement humaines. Certes, les deux versions font appel à l'image de la roue de Fortune ${ }^{27}$, mais elle représente, chez l'un, la providence divine, chez l'autre, le hasard aveugle.

Ces divergences se répercutent jusque dans l'évocation des Turcs. Si l'on s'accorde à en brosser un portrait négatif, les griefs formulés ne reposent pas toujours sur les mêmes critères. Michel Pintoin condamne leur identité religieuse, comme s'il

\footnotetext{
${ }^{24}$ Etudiant les complaintes du Religieux de Saint-Denis, B. Guenée montre qu'elles s'inspirent souvent du Tragicum argumentum de miserabili statu regni Francie, que François de Montebelluna avait écrit en 1357 pour prouver, après la défaite de Poitiers, que Dieu avait justement châtié les Français de leurs péchés (art. cit.). Il n'est pas impossible que le style déclamatoire adopté par Michel Pintoin dans l'épisode de Nicopolis obéisse au même dessein.

${ }^{25}$ Pour l'historique de cette idée, voir C. Beaune, Naissance de la nation France, Paris, Gallimard, 1985 (Bibliothèque des Histoires), chap.VII.

${ }^{26}$ Chron., XXVII, 510. L'idée de la colère divine revient à plusieurs endroits : cf. XXIV, 496 (non digni gracia sunt inventi) et XXV, 502.

${ }^{27}$ Chron., XXVI, 508 ; Bouc., XXIV, 99.
} 
cherchait, en inscrivant les Turcs au nombre des agents de Satan, à réveiller la chrétienté et à reconstituer l'unité détruite par le Schisme. L'islam est à ses yeux synonyme de souillure (regiones spiritu Mahometis pollutas, XXIII, 484), de sacrilège (Turcorum manus sacrilegas, XXVII, 512) et de blasphème (blasphemantibus nomen [Domini], XXIV, 496). Pourtant, Bajazet bénéficie de circonstances atténuantes : face aux chrétiens débauchés et indisciplinés, il fait figure de sage (vir providus et discretus, ibid., 498). Bien vite, on s'aperçoit que l'ambiguïté fragilise les frontières qui séparent les deux mondes antagoniques. Français et Turcs partagent les mêmes vices, cruauté, impureté... On croirait presque entendre les propos hérétiques de l'Anglais John Wycliffe (1324-1384), qui confondait dans une même réprobation l'islam et l'Eglise européenne : «Nous sommes les Mahomets de l'Occident» ${ }^{28}$... Plus vraisemblablement, le motif de la souillure est à rattacher à l'Epistre lamentable et consolatoire (1397), où Philippe de Mézières interprète les désordres sociopolitiques en termes de maladie et de médecine, comparant la défaite de Nicopolis à l'effet d'un déséquilibre humoral, d'une corruption des humeurs nécessaires à la bonne santé ${ }^{29}$. Le Religieux de Saint-Denis n'en appelle-t-il pas, lui aussi, à une réforme morale, régénératrice? Or le biographe de Boucicaut, lui, dresse une évocation plus stéréotypée de l'ennemi, qu'il qualifie volontiers de «Sarrasins», alors que Michel Pintoin préfère parler de Turci. On reconnaît, dans le Livre des faits, les caractéristiques des païens décrits dans les chansons de geste : la "malice» $(\mathrm{XXV}, 106)$ et l'usage d'armes non réglementaires, en particulier ces «maçues de cuivre» (ibid., 109) qui rappellent l'attribut du fou et du vilain, et qui deviennent, lors du martyre des chrétiens, des couteaux de bouchers (XXVI, 115). La campagne de Nicopolis prend l'aspect d'une croisade épique, avec ses traîtres (Sigismond) et sa «chiennaille de mescreans"...

Mais c'est à la fin de l'épisode que l'écart idéologique atteint son amplitude maximale. Michel Pintoin ne parle pas du retour des croisés, alors que le biographe décrit les manifestations de joie qui accueillirent, en France, le comte de Nevers et les autres rescapés ${ }^{30}$. Le silence méprisant du Religieux de Saint-Denis s'inscrit dans le cadre de son argumentation théologique : le récit s'achève sur le martyre glorieux des chrétiens. Evoquer le sort des survivants aurait nui à la cohérence du message salvateur: «Pas question des retours, dans la croisade. La croisade est un pèlerinage sans retour. Jérusalem et la gloire..., après quoi, il n'y a plus d'après. Quand le retour se fera selon la gloire des hommes, c'est qu'aura manqué à Jérusalem, par la faute des soldats de la Croix..., la manifestation parousique de Dieu» ${ }^{31}$. C'est peut-être la nostalgie de ces croisades passées qui explique, dans la Chronique, l'inachèvement de l'épisode de Nicopolis. A peine y est-il question de la douleur soulevée à Paris par l'annonce du désastre : Michel Pintoin insiste sur le coup de théâtre provoqué par les messagers, brisant la joie d'une paix imminente entre la France et l'Ángleterre, et sur la tonalité chrétienne des lamentations, consacrées à l'indigne sépulture des

\footnotetext{
${ }^{28}$ Cité par Ph. Sénac, L'Image de l'autre. Histoire de l'Occident médiéval face à l'islam, Paris, Flammarion, 1983, p. 141.

${ }^{29} \mathrm{~J}$.-L. Picherit, La métaphore pathologique et thérapeutique à la fin du Moyen Age, Tübingen, Niemeyer, 1994 (Beihefte zur Zeitschrift für romanische Philologie, 260), chap.V.

${ }^{30}$ Bouc., XXVIII, 128.

${ }^{31}$ A. Dupront, op. cit, p. 263.
} 
chrétiens morts en Hongrie ${ }^{32}$. Ces deux notations, qui placent l'événement au carrefour du temporel et du spirituel, focalisent l'éclairage sur le destin du royaume. A l'inverse, c'est un deuil exclusivement privé et fortement socialisé qui retient l'attention du biographe de Boucicaut : la douleur des veuves, énumérées une par une, s'accorde à l'idéologie courtoise de son récit, et l'esprit de caste domine la déploration funèbre, où «chacun plaignoit la noble chevalerie, qui estoit comme la fleur de France» (XXVII, 119).

Quant au facteur financier, de plus en plus important dans l'organisation des campagnes militaires aux $X I V^{e}$ et $X V^{e}$ siècles, on n'en trouve aucune trace chez le Religieux de Saint-Denis. L'enjeu économique des rançons négociées entre les chrétiens et Bajazet n'entre pas en compte dans ce récit orienté vers une sacralisation de la défaite. Selon Michel Pintoin, le sultan n'aurait épargné la vie du comte de Nevers que par désir de l'humilier davantage, lui et la foi chrétienne (ejus displicencia et ignominia fidei christiane, XXVIII, 516), en l'obligeant à assister au sacrifice des autres prisonniers. Or dans la version du biographe, on apprend que cette grâce, étendue à tous les «parens prochains du roy de France», tendait à des fins plus matérielles, à savoir le «tres grant tresor et finance» que Bajazet entendait soutirer de si précieux captifs (XXVI, 114). Le sultan s'avère insatiable de richesses, et seul l'envoi de "moult riches et beaulx presens» (XXVII, 120) ou la promesse de «mains beaulx dons» (XXVIII, 124) peuvent fléchir sa colère. Comme pour détourner l'accusation lancée contre les seigneurs occidentaux soupçonnés de voir dans l'Orient un bien de consommation et non plus un lieu de conflit spirituel, le biographe nous montre l'étonnement des Turcs éblouis par les trésors de l'Occident, «dont ilz n'ont mie par dela» (ibid., 127). En privilégiant le facteur économique, et notamment la participation personnelle de Boucicaut aux frais de l'expédition («y mena a ses despens .LXX. gentilz hommes», XXII, 91), il inscrit la croisade au nombre des obligations chevaleresques. Elle fonctionne pour l'aristocratie française comme un test social, l'occasion de manifester non seulement sa prouesse mais sa largesse ${ }^{33}$.

Ces ambitions didactiques reposent, chez nos deux auteurs, sur la mise en place d'une écriture persuasive. Les références à l'Histoire Sainte, en particulier, offrent l'avantage de promouvoir l'actualité à la hauteur du sacré ancien. Ainsi, le martyre des chrétiens est comparé au massacre des Innocents dans la version du biographe ${ }^{34}$, et Michel Pintoin semble suggérer le même rapprochement en parlant d'un Dieu qui «laisse châtier ceux de ses enfants qu'il a jugé dignes de recevoir dans son royaume» (permittit filios flagellari, quos recipiendos dignos duxit, XXVIII, 518). Mais, dans le récit même de la bataille, le Livre des faits compte peu d'allusions bibliques : seule la fuite des Hongrois rappelle l'abandon du Christ, qui «fu delaissié de sa gent si tost que il fu es mains de ses ennemis» (XXV, 107). Fidèle à la tradition héroïque, le biographe préfère recourir aux comparaisons épiques pour décrire l'ardeur des Français, acharnés à se défendre comme «cenglier escumant, loup enragié, ou lyons forcenez» (ibid., 108 et 113). Le Religieux de Saint-Denis, au contraire, ponctue son

\footnotetext{
${ }^{32}$ Chron., XXIX, 522.

${ }^{33}$ Sur l'importance de la croisade dans la culture chevaleresque, voir N. Housley, op. cit., pp. 395-397, et H. Platelle, Les Croisades, Desclée, 1994 (Bibliothèque d'Histoire du christianisme, 33), pp. 166-167.

${ }^{34}$ Bouc., XXVI, 114.
} 
récit de commentaires tirés des livres sapientiaux. Ainsi, lorsque les chrétiens, apercevant les troupes de Bajazet, regrettent leur témérité, il glisse un verset tiré du Livre des Proverbes: «l'impie s'enfuit, même sans qu'on le poursuive» (fugit enim impius ..., nemine persequente, XXVII, 510 ${ }^{35}$. C'est aussi une image biblique qu'il emprunte pour condamner l'obstination des juvenes sourds aux conseils de leurs aînés, «comme l'aspic insensible qui se bouche les oreilles» (velut aspis surda obturans aures suas, XXIV, 494) ${ }^{36}$. Enfin, la parole des prophètes a plus de poids que celle de l'historien pour enseigner la soumission chrétienne aux desseins de Dieu (judicia tua abyssus multa, XXVII, 511) ${ }^{37}$. Parfois, Michel Pintoin choisit parmi les acteurs de Nicopolis les interprètes de cette sagesse gnomique. Le conflit de générations qui sape l'unité des forces alliées est ainsi résumé de manière lapidaire par le roi de Hongrie, pour qui «la jeunesse a plus d'ardeur, mais la vieillesse plus de prudence» (major sit juventutis fervor, major tamen gravitas senectutis, XXIV, 494). Bajazet lui-même cite Valère-Maxime : «la colère divine marche d'un pas lent à la vengeance» (lento gradu ad vindictam divina procedit ira, XXIV, 498). On voit donc, par le choix des citations, que l'épisode de Nicopolis a subi un traitement littéraire, à coloration épique chez le biographe de Boucicaut, plus nettement biblique chez le Religieux de Saint-Denis.

La défaite de Nicopolis illustre la puissance narratogénique du malheur : l'existence paisible des gens heureux n'intéresse personne, tandis que les tribulations humaines inspirent romanciers et historiens. Ni Michel Pintoin ni le panégyriste de Boucicaut n'ont pu contenir leur exaltation littéraire, au souvenir du massacre de 1396. Par-delà le chroniqueur et le biographe, nous devinons des écrivains conscients de leur art, et de leur responsabilité dans un débat aussi agité que celui de Nicopolis. Leurs témoignages laissent entendre l'émotion que suscite encore l'idée de croisade, à une époque où l'indifférence gagne du terrain. A défaut d'éclairer la vérité événementielle, c'est un autre combat, verbal celui-ci, qu'ils livrent au jugement de la postérité. Faut-il s'en plaindre? «Immense armée des fils d'Eole, nés du vent et glonflés de mots. Ils soufflèrent. A leur souffle, une Babel de mensonges et de billevesées, un solide brouillard, magiquement épaissi, où la raison ne mordait pas, s'éleva dans les airs. L'humanité s'assit au pied, morne, silencieuse, renonçant à la Vérité» ${ }^{38}$... Or n'y a-t-il pas quelque naïveté à supposer chez tout homme un instinct qui le pousserait à connaître la Vérité ?

\section{Elisabeth Gaucher}

\footnotetext{
${ }^{35}$ Proverbes, 28, 1 : «Le méchant s'enfuit quand nul ne le poursuit, d'un lionceau les justes ont l'assurance». Michel Pintoin attribue aux chrétiens la marque de couardise, et non aux infidèles.

${ }^{36}$ Psaumes, 57, 5. Nous devons cette identification à B. Guenée (introduction à l'éd. cit., p. LIX), qui cite aussi le proverbe asino surdo narrare fabulam (XXIII, 484).

${ }^{37}$ Isaïe, 40, 28 («insondable est ton intelligence») ; cf. Epitre aux Romains, 11, 33, et Psaumes, 139, 17.

${ }^{38}$ J. Michelet, L'agonie du Moyen Age, Editions Complexes, 1990, pp. 124-25.
} 